\title{
Slab bridges with arbitrary shape and support conditions: a general method of analysis based on finite elements
}

\author{
Y. K. CHEUNG, I. P. KING \& O. C. ZIENKIEWICZ
}

\section{Professor F. Sawko, Department of Civil Engineering, University of Liverpool}

The Authors have over the past few years made a substantial contribution to the subject of finite element methods and this Paper is a logical use of the basic triangular element derived three years $\mathrm{ago}^{7}$ for the analysis of slabs with variable geometry and boundary conditions. I have used the finite element method for the analysis of various bridge structures ${ }^{20-22}$ and should like to endorse the advantages claimed for this approach.

97. Finite elements have become fashionable and it is common to hear 'Why not use the finite element method?' I generally reply that the method is only practicable when fully automatic computer programs are developed in conjunction with the particular elements. On the basis of this Paper the Authors can justly claim that this is indeed the case for isotropic slab bridges. The problem of orthotropic bridges, however, has to be examined far more carefully before a general claim can be extended to these structures.

98. Orthotropic slab bridges are of two types:

(a) reinforced concrete slabs (assumed cracked) with different amounts of reinforcement;

(b) cored or cellular decks.

The first alternative lends itself to analysis by the Authors' program, since the triangular element is capable of simulating any two dimensional orthotropy. Cored slabs cannot be analysed using the existing program, and I disagree with several statements and claims made in $\$ 62$ and 63 .

99. The Authors say that four constants are required for defining the properties of perforated or cellular slabs (Fig. 11) quoting Timoshenko and WoinowskyKrieger. ${ }^{11}$ The four constants referred to are the flexural stiffnesses (EI) in longitudinal and transverse directions and the corresponding torsional stiffnesses (GJ). In my opinion a fifth constant is required, defining the shear flexibility of the transverse medium. The medium consists of an effective beam with cut-outs in the web and resembles a vierendeel girder (or a single bay multi-storey frame) in appearance and action. It is easy to visualize a multi-storey frame swaying under lateral loading, suggesting that a large proportion of total deflexion is due to flexure of individual columns and beams; indeed, the usual assumption of neglecting axial strains in members is tantamount to assuming that all the deflexion is due to shear effects.

100. It is obvious that the relative importance of shear versus flexural effects of the transverse medium is a function of the size and shape of openings. This effect was investigated two years $\mathbf{a g o}^{23.24}$ and Figs 17 and 18 summarize the results. Several perspex beams with different ratios of hole size to depth were tested under a four point loading (Fig. 17) and the relative importance of shear against bending deformation is shown in Fig. 18. For the shape of cells considered and for values of $d / a$ commonly found in bridge decks the shear deformations were found to be dominant.

Paper published: Proc. Instn civ. Engrs, 1968, 40 (May) 9-36. 


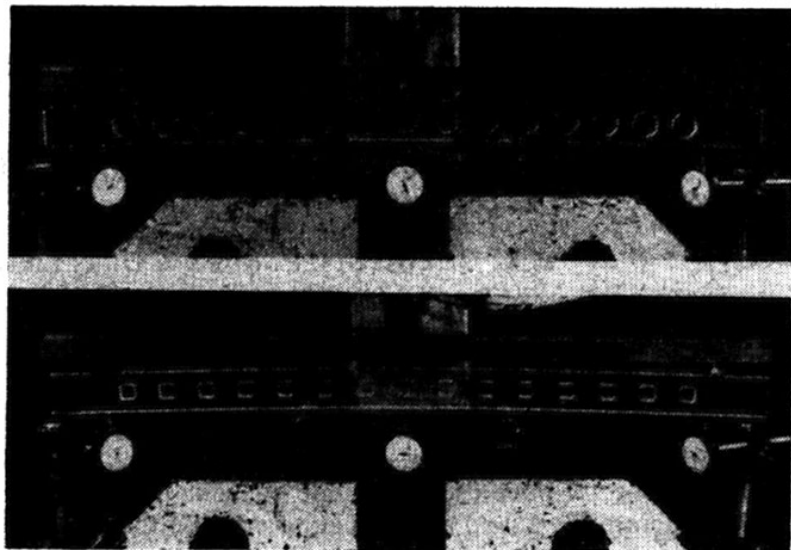

Fig. 17 (above). Perspex beams with circular and square openings under test

Fig. 18 (below). Effect of shear effects on deformation of beams with openings

Fig. 19 (opposite). Comparison of experimental and grillage analysis of Cumberland Basin slab (Cement and Concrete Association)

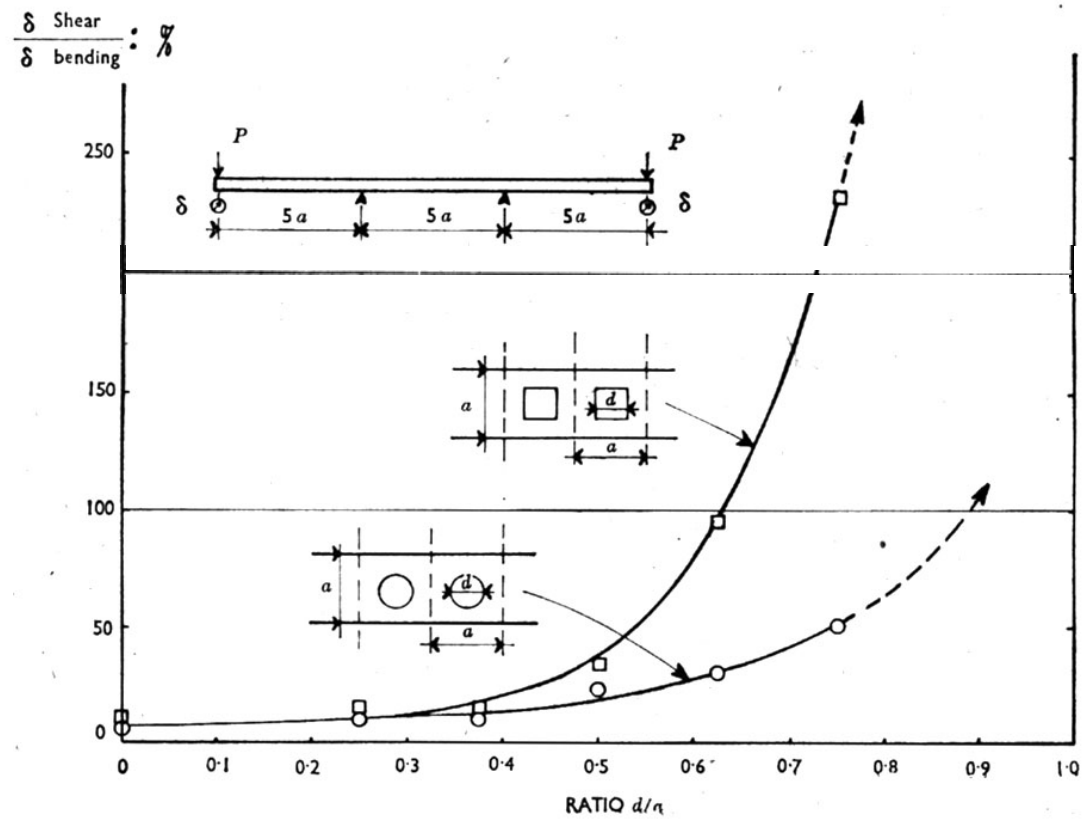


101. These tests and others show that shear deformation of transverse media cannot be neglected in a two dimensional idealization. They also show that square holes exhibit a far greater shear flexibility than circular holes (because of the haunch effect in circular openings) and that longitudinal rectangular openings (smaller dimensions being within the depth of slab) are more flexible in shear than square voids. All three types of voids are currently used in design.

102. I found a simple model test very effective in deriving the two transverse parameters, but a two dimensional plane strain analysis (using finite elements) would be equally effective. The Authors' suggestion of a three dimensional analysis $(\$ 63)$ seems unnecessary and logically inconsistent with two dimensional idealization of cellular slabs.

103. Once this effect of shear flexibility of the transverse medium is recognized, there is no basic difficulty in modifying existing methods of analysis to incorporate the effect. I have incorporated this facility in my grillage program ${ }^{22}$ and shown that good accuracy can be obtained using single member idealization of the vierendeel effect. The Authors' triangular and rectangular elements are now being modified by a research student to allow for this shear flexibility effect. Once this work is complete, it will be possible to assess numerically the importance of shear deformations in cored slabs and to use actual geometric parameters for any bridge analysis.

104. I feel that the Authors have dismissed the grillage approach ( $\$ 9$ and 10 ) too lightly. I have used it for the past nine years in the analysis of several complex bridge structures. Its accuracy for right bridge decks has been shown ${ }^{25}$ and recently the Cement and Concrete Association used the grillage analysis for the Cumberland Basin slab (which has complex random support conditions) and Fig. 19 shows the excellent correlation between experimental and grillage results. I should be interested in a comparison of relative accuracies of the two numerical approaches for the same computational effort (i.e. the same number of nodes) for this slab.

105. The Authors also imply that varying section slabs can be similarly treated by the uniform triangular element. This approach will present many difficulties since elements of different thicknesses will be assumed jointed at nodes and my research team are developing varying section elements for these structures.

106. The final word of warning contained in $\S 81$ is very appropriate, and there is a danger that this enthusiasm the Authors have for finite elements (which I share) might lead engineers to an indiscriminate use of this powerful trend. In the field of bridge analysis not all the problems have been solved yet.

\section{Dr A. R. Baker and Mr C. M. MacMillan, Ove Arup \& Partners}

The reaction results quoted for the Cumberland Basin Bridge are interesting in that the experimental results showed a shedding of load from the most heavily loaded columns towards the more lightly loaded when compared with the analytical results.

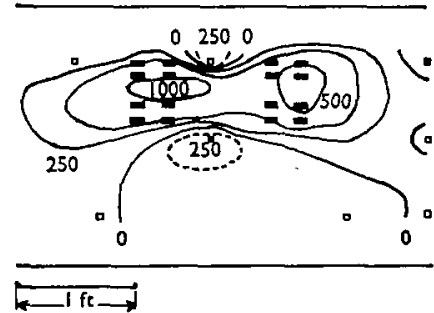

Slab model-measured stresses

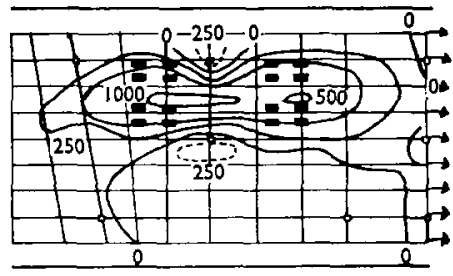

Simulated grillage-computed stresses

TRANSVERSE STRESSES DUE TO H.B. LOAD IN POSITION SHOWN 
Table 3. Cumberland Basin bridge-support reactions

\begin{tabular}{c|c|c|c}
\hline $\begin{array}{c}\text { Column } \\
\text { number }\end{array}$ & Experimental & $\begin{array}{c}\text { Rectangular finite } \\
\text { elements } \\
\text { with springs }\end{array}$ & $\begin{array}{c}\text { Finite element mesh } \\
\text { in Fig. 8(b) } \\
\text { rigid supports }\end{array}$ \\
\hline 1 & 675 & 721 & 632 \\
2 & 1495 & 1550 & 1718 \\
3 & 750 & 741 & 666 \\
\hline 4 & 1360 & 1342 & 1230 \\
5 & 2025 & 2180 & 2450 \\
\hline 7 & 955 & 817 & 829 \\
\hline 8 & 935 & 858 & 696 \\
\hline 9 & 832 & 829 & 613 \\
\hline 10 & 1078 & 2257 & 2626 \\
\hline
\end{tabular}

This clearly suggested support flexibility in the model. Accordingly we analysed the bridge as if supported on elastic columns.

108. The analysis was made using a computer program based on a rectangular finite element. ${ }^{26}$ The approach has been found to give good results using a comparatively coarse mesh.

109. A $6 \times 20$ mesh similar to the one shown in Fig. 8(a) was used. The elastic columns were presumed to have a vertical stiffness of $209000 \mathrm{Jb} / \mathrm{in}$., a figure suggested as realistic by Best and West describing the experimental work. ${ }^{27}$ Fully consistent nodal forces were used to represent the distributed load.

110. The results obtained for the reactions are shown in Table 3.

111. The closer agreement with the experimental results serves to re-emphasize the importance of attempting to represent the actual flexibility of supports. In practice the determination of realistic deformation characteristics for such supports is extremely difficult as they would almost certainly be highly non-linear and time dependent.

112. The Authors have mentioned the facility for including beam elements in their program. However, the stiffening beams are normally eccentric to the slab and, as the Authors have stated, a three dimensional analysis is necessary to simulate the action fully since the slab is subject to in-plane and bending stresses.

113. To reduce the problem to a two dimensional one therefore involves approximating to the stiffness constants for the beam element. For a down-stand beam, for instance, they would certainly exceed those for the rib section alone. Constants appropriate to a T-beam involving some arbitrary width of the slab would presumably be more realistic. Have the Authors done any work from which they could make recommendations about this?

114. The cellular slabs in Fig. 11 are referred to as 'highly anisotropic'. From some tests performed on perspex models this is contrary to the experience we have had. It seems difficult to produce an in situ concrete cellular section for which the orthotropic stiffness constants differ by more than $25 \%$. This would suggest that in many instances the refinement of an orthotropic slab analysis is not consistent with the other approximations inherent in the analysis.

Mr K. Kretsis, Stressed Concrete Design, Ltd

Having been involved in the design of the Castleton bridge mentioned in $\$ 73-77$ I should like to make the following observations. 
116. In Fig. 14 it is shown that the real cross section (a), where the edge beams are completely above the slab with the neutral plane of the beams themselves above that of the slab, has been idealized as in (b) with a common neutral plane but a greater beam depth. The greater beam depth will compensate for the stiffness of the composite $L$ section of the real structure, so that the vertical shear forces between the slab and the edge beams will probably be evaluated correctly, but the idealized cross section does not bring into the picture the longitudinal shears which also develop at the joints through the in-plane actions of the slab. This latter aspect has a considerable effect, particularly in narrow bridges where the whole structure will act as a kind of channel or only near the edges of wide structures. In the case of Castleton bridge the stresses in the slab near the joints were not equal to those at the bottom of the edge beams if the equivalent $\mathbf{L}$ section could not be assessed by other means.

117. I would therefore suggest that the introduction of the in-plane actions of the slab would be a worthwhile improvement of this program, perhaps by simply adding to the flexural stiffness matrices of the elements their already existing stiffness matrices for two dimensional in-plane actions. It is obvious that this will have to be combined with the addition of three more displacements at each joint, namely $W_{x}, W_{y}$ and $\theta_{z}$ to the already assumed $W_{z}, \theta_{x}, \theta_{y}$, thus doubling the number of unknowns.

118. Could the Authors comment on the suggestion and is such an extension likely in the near future with the storage capacity of existing computers?

\section{Dr W. M. Jenkins and Mr D. J. Lax, University of Bradford}

The Authors have adopted a triangular flexural element as being the most suitable element for the analysis of slab bridges of arbitrary shape. In dealing with the problems associated with columns of finite area the triangular element is well suited to localized mesh refinement. If a rectangular or parallelogrammic element is used it is not possible to carry out mesh refinement around the columns without considerably increasing the total number of nodes throughout the structure. If the column area is represented by a single, rigid element this also governs the size of adjoining elements. To get over this difficulty in the use of rectangular and parallelogrammic elements we have developed a special element which incorporates a rigid, rectangular region in one corner. The use of such an element avoids the need for mesh refinement and allows the analysis to proceed using a coarse division into elements if desired.

120. The element is shown in. Fig. 20 with the rigid column area shaded. Node 1 lies on the column axis, the total column area being shared between the adjacent elements. The element stiffness matrix is evaluated using the standard procedure ${ }^{\mathfrak{B}}$ in the form:

$$
K=\left(C^{-1}\right)^{T} \iint B^{T} D B d x d y C^{-1}
$$

in which the double integration is made over the unshaded area in Fig. 20. In the subsequent structural analysis the material represented by the shaded area behaves as a rigid body subjected to rotations $\theta_{x}, \theta_{y}$ and transverse deflexions $w$ at node 1 .

121. The element has been used to analyse the problem shown in Fig. 4 and the results are shown by the dotted line in Fig. 21. Evidently there is good agreement with the exact solution, particularly as to the position and value of the maximum moment.

122. In describing the three types of element in $\S 20$, the Authors include a beam element with two nodes lying in the plane of the slab. We are currently investigating problems associated with slab-beam interaction in bridges in which the centroidal axis of the beam lies at some distance below the slab. We have concluded that, in order to represent the slab and beam by different elements, account must be taken of the in-plane forces in both. Strictly a three dimensional analysis is required and the demands on computer space and time will be heavy. An acceptable approximation for some bridges might be to find a neutral plane for the bridge and locate the nodes in this plane. The two dimensional approach may then be retained and three degrees 


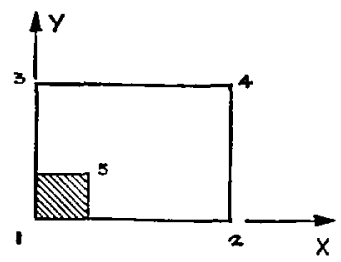

Fig. 20 (left)

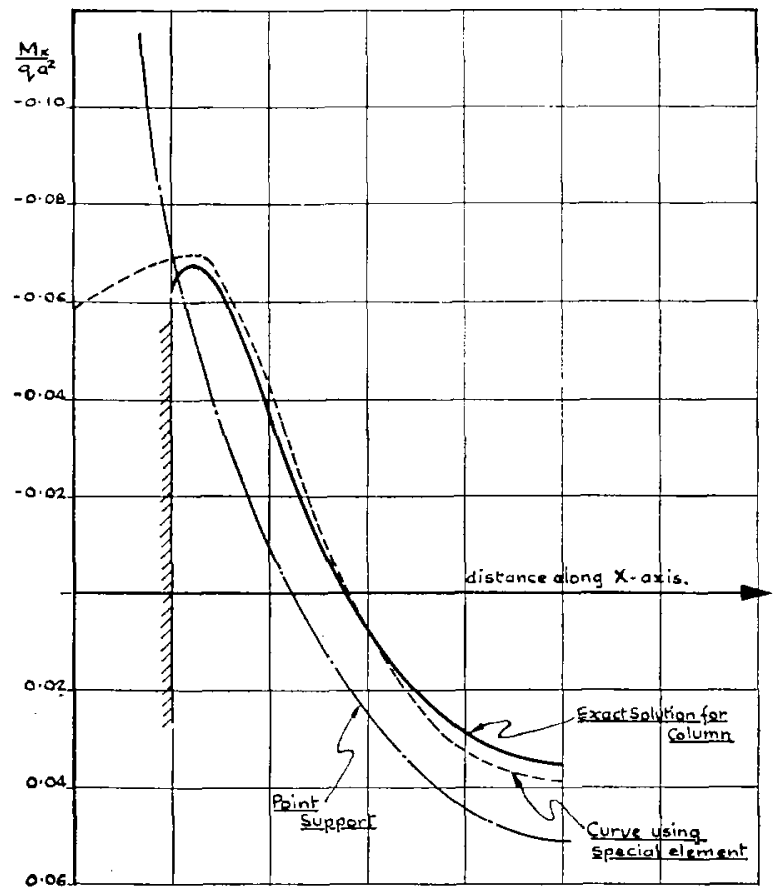

of freedom defined at each node. The individual element stiffnesses, including inplane effects, are then appropriately transformed and the global stiffness matrix assembled.

Mr B. J. Thorpe, Howard Humphreys and Sons

The Authors are to be congratulated on the considerable advances which they have made in this method of analysis. The bridge designer is now presented with a means of determining the behaviour of complex structures which previously either were difficult to deal with because of unadaptable analytical procedures, or else required a model test involving delays and high cost.

124. The suggestion that the differences which are noted when comparing results obtained by finite element analysis with those from model tests may be due to errors inherent in the model testing is not unreasonable, but the agreement obtained in the examples cited in the Paper demonstrates that where a suitable finite element mesh has been used either set of results is generally good enough for practical engineering purposes. 
125. The Authors' 'word of warning ... . lest indiscriminate use of powerful techniques leads the engineer "up the garden path" should, however, be heeded. Although the examples given in the Paper yield a wealth of information regarding the types of mesh which can prove suitable, the exact choice of mesh size and configuration for a particular problem can present difficulty.

126. In our own use of the Authors' finite element program for the analysis of a skewed, prestressed in situ concrete slab, continuous over five supports, refinement of the mesh size in the neighbourhood of the supports to 'pick-up' the behaviour of the slab around the bearings produced results tending towards the predictable singularities. However, the real structure cannot be considered to be a thin plate at the supports and a 'rounding off' of the moment diagrams is obviously permissible.

127. Similarly, where bearings are closely spaced the assumption of a line support condition under the thin plate analysed, with no bending occurring along the line of the support, may be a more realistic representation of the behaviour of the structure than the singularities produced by inserting discrete point supports at each bearing.

128. It is for cases such as these that the experience gained from the three dimensional finite element approach might yield guidance in the selection of a suitable mesh size and configuration which could yield reasonable results direct from the two dimensional analysis without the need for a costly three dimensional study. Are the Authors intending to publish results of their investigations into such 'local effects' and do these investigations cover the situations which commonly occur in practice in bridgeworks?

\section{Mr C. J. Parekh and Mr R. G. Anderson, University of Wales, Swansea}

The Authors have produced and assessed a method of analysing slab bridges using a digital computer. The program developed has been successfully tested against exact and experimental solutions and presents an extremely versatile and economic approach to such problems. The specifications of the program BAPS ${ }^{2 \theta}$ are now available and are designed for ease of use.

130. With the development of sophisticated computer programs for complex structures, emphasis should be placed upon the 'input' and 'output' aspects of the problems. The amount of data that has to be prepared and checked for many programs is excessive and time consuming. The amount of results produced is enormous. A program should be efficiently designed to keep the volume of data to a minimum. To this end programs have been developed to automate the procedure for preparing and handling the data. Program IPUT ${ }^{20}$ has been written to accept simplified data to define the problem, for example, geometry of the bridge, material properties, boundary conditions and loads. IPUT generates all the input to be executed directly with BAPS but is restricted to a bridge with parallel edges which are straight or curved (circular or transition curve) in plan. Program OPUT ${ }^{30}$ processes the results and will carry out any desired combinations. It outputs the maximum and minimum values of the bending moments of any number of specified points thus enabling the user to control the volume of output required. OPUT can easily be adopted to calculate the design moments for reinforcements. Examples of the use of graphic output in the form of vector and contour plots are given in the Paper.

131. In the analysis of slab bridges it is often desirable to know, in addition to the bending moments, the distribution of shear forces over the slab. It was therefore thought necessary to modify BAPS to evaluate the shear forces.

132. The formulation to evaluate the shear forces is similar to the procedure used to find the bending moments in the Paper.

133. Using identical notations the shear forces are defined as

where for a plate element

$$
\{\sigma\}_{s}=[D]_{s}\{\epsilon\}_{s}
$$

$$
\{\sigma\}_{s}=\left\{\begin{array}{l}
Q_{x} \\
Q_{y}
\end{array}\right\}
$$


DISCUSSION

is the vector of shear forces, and

$$
[D]_{\mathrm{s}}=\left[\begin{array}{cc}
D_{x} & 0 \\
0 & D_{y}
\end{array}\right]
$$

is the elasticity matrix for an orthotropic plate. Finally

is the shear vector and

$$
\left\{\epsilon_{s}=\left\{\begin{array}{c}
-\frac{\partial}{\partial x}(\nabla w) \\
-\frac{\partial}{\partial y}(\nabla w)
\end{array}\right\}\right.
$$

$$
\nabla w=\frac{\partial^{2} w}{\partial x^{2}}+\frac{\partial^{2} w}{\partial y^{2}}
$$

Substituting for $w$ in terms of the shape functions for the triangular plate bending element used in the Paper (see also ref. 31, p. 100 et seq.) reduces equation (3) to

$$
\{\sigma\}_{\mathrm{s}}=[D]_{\mathrm{s}}[B]_{\mathrm{s}}[T]\left\{\delta^{\mathrm{e}}\right\} \quad \cdot \cdot \cdot \cdot \cdot \cdot \cdot
$$

where $T$ and $\left\{\delta^{\theta}\right\}$ were defined in the Paper and $[B]$ is easily formulated.

134. The procedure to evaluate shear forces for beam elements is identical and in fact much more straightforward.

135. The comments in the Paper under the section on moment 'averaging' will also apply to the shear force. The shear values are computed only for the centroid

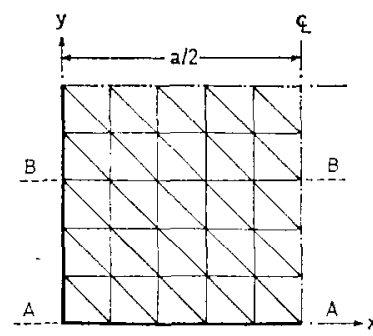

$5 \times 5$ mesh

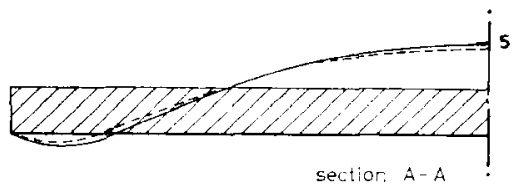

(a)

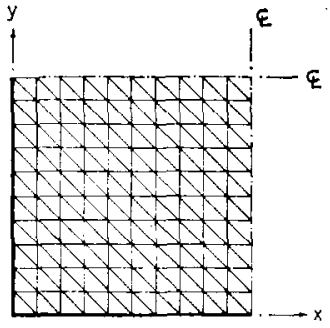

$10 \times 10$ mesh
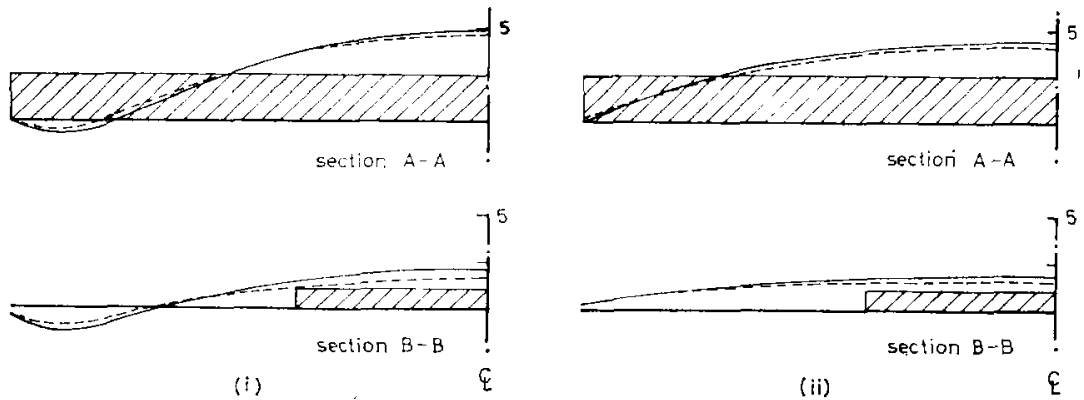

(b)

Fig. 22. Square plate under uniform load : (a) finite element idealization; (b) distribution of $Q_{y}$ on sections $A-A$ and $B-B$; (i) square plate with all edges built in ; (ii) square plate with all edges simply supported $10 \times 10$ mesh

$-----5 \times 5$ mesh 
of each triangular element and are subsequently averaged at the nodal points. For beam elements the shear forces are computed at the nodal points.

136. To assess the general accuracy of shear forces in plates by the triangular plate element, the problem of a uniformly loaded square plate under different support conditions was tested for two sub-divisions.

137. Due to the symmetry of the problem chosen it is necessary to solve only a quarter of the plate. The sub-divisions used are shown in Fig. 22(a).

138. Solutions were made for three different boundary conditions. Some results obtained for a plate with all sides built in and for a plate with all sides simply supported are shown in Figs 22(b) (i) and 22(b) (ii) respectively. The cross hatched areas represent the total shear to be carried at the respective sections, while the solid line represents the shear calculated for the $10 \times 10$ mesh and the dotted lines those for the $5 \times 5$ mesh. As expected the increase in mesh size gives an improved result.

139. In addition to the two boundary conditions mentioned a further solution was made for a plate built in on two opposite edges and free on the other two edges. By integrating the shears over a particular section it is possible to compare the total computed against the actual shear force. This process was carried out for sections $\mathbf{A}-\mathbf{A}$ and $\mathbf{B}-\mathbf{B}$. The accuracies obtained varied considerably, but in the area maximum shear at section $\mathrm{A}-\mathrm{A}$ were never more than $20 \%$ different from the expected value. However, much greater discrepancies occurred at section B-B. In each case the finite element result proved to be an over estimation of the total shear.

140. The shape function used for the triangular plate bending element is not of sufficiently high an order to give a very meaningful shear acting over a single element. The shear is in fact a constant value over each element. It was therefore necessary to average the shear at each nodal point to obtain the results quoted.

141. From the results obtained and knowledge of the element used it must be stressed that the shear values obtained by such an analysis only give the engineer an idea of how the shear acts within a certain plate and should be treated with suspicion.

142. As a typical application of the formulation given here, the curved bridge deck with the hinge described in the Paper (Fig. 9) was analysed. The geometry and loading conditions were identical. Computer plots of shear force contours are shown in Fig. 23.

143. The times taken to analyse the curved bridge deck with 228 elements, 140 nodal points and 420 unknown were $8 \mathrm{~min} 15 \mathrm{~s}$ and $14 \mathrm{~min} 40 \mathrm{~s}$ with and without evaluating shear forces respectively. The times quoted are for ICT 1905 computer.

\section{Mr B. C. Best and Mr R. West, Cement and Concrete Association}

We should like to comment on the comparisons made by the Authors between the results obtained by the two theoretical analyses and the model analysis of the Cumberland Basin scheme. The two theoretical analyses show reasonable agreement as they are attempting to deal with the same structure; the assumptions made for boundary conditions, however, are not those existing in the model.

145. The model was supported on a composite column formed from five main elements. When loaded these columns underwent an elastic shortening considerably in excess of the sum of the individual main elements. If the column reactions in Fig. 8(a) are examined, it will be seen that for the centre line of columns the model results are lower than from the theory and for the two outer lines of columns this is reversed. This is obviously due to shedding of the load by the more heavily loaded columns. This effect is also marked when studying the contours for distribution of bending moments.

146. Since the completion of testing of the model the slab has been analysed under various loading configurations using a rectangular finite element and also using a grid idealization, in each case assuming both rigid supports and elastic supports. For both idealizations the analyses for an elastically supported slab are comparable with the behaviour of the model. 


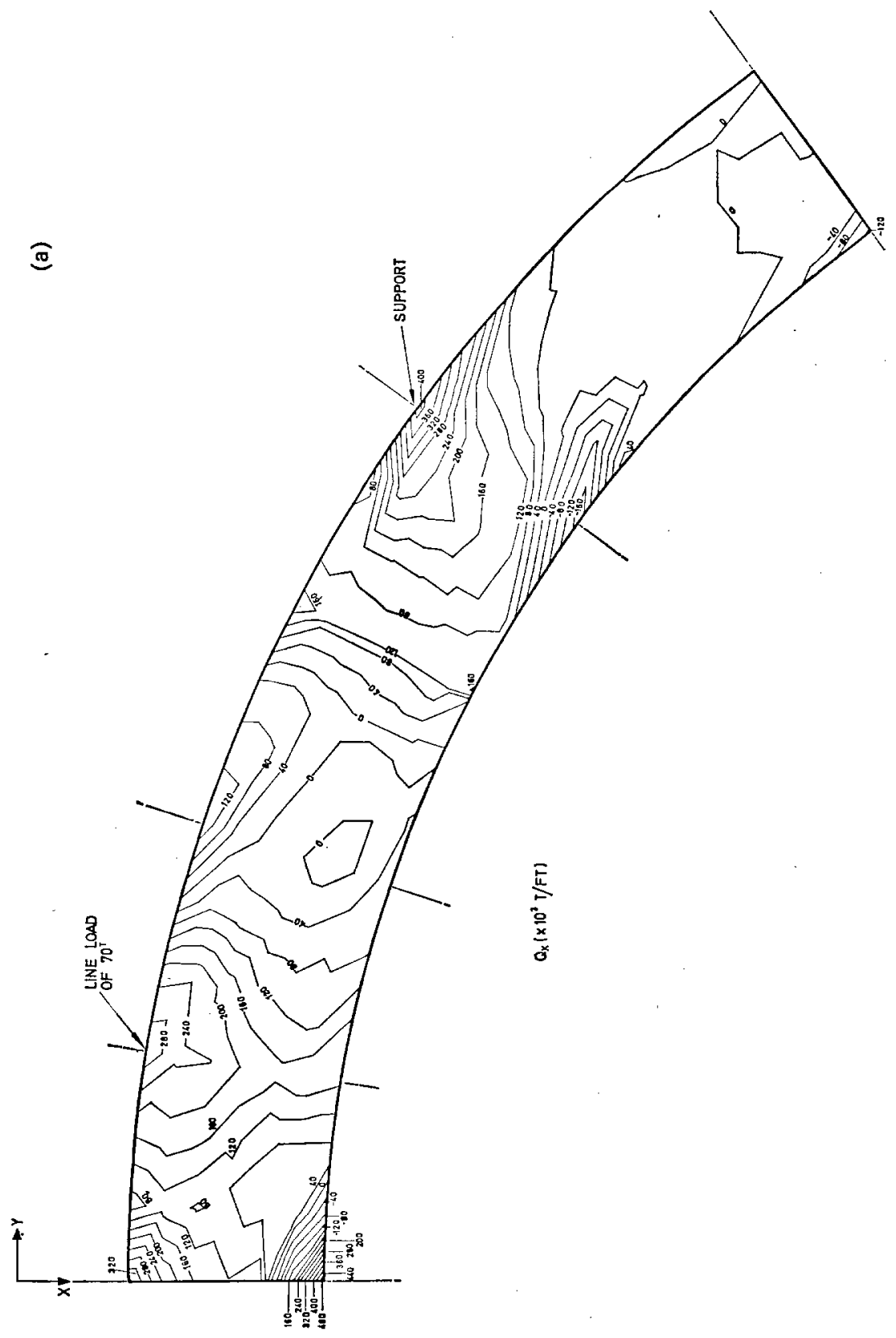

822

Downloaded by [] on [26/04/23]. Copyright ( ) ICE Publishing, all rights reserved. 


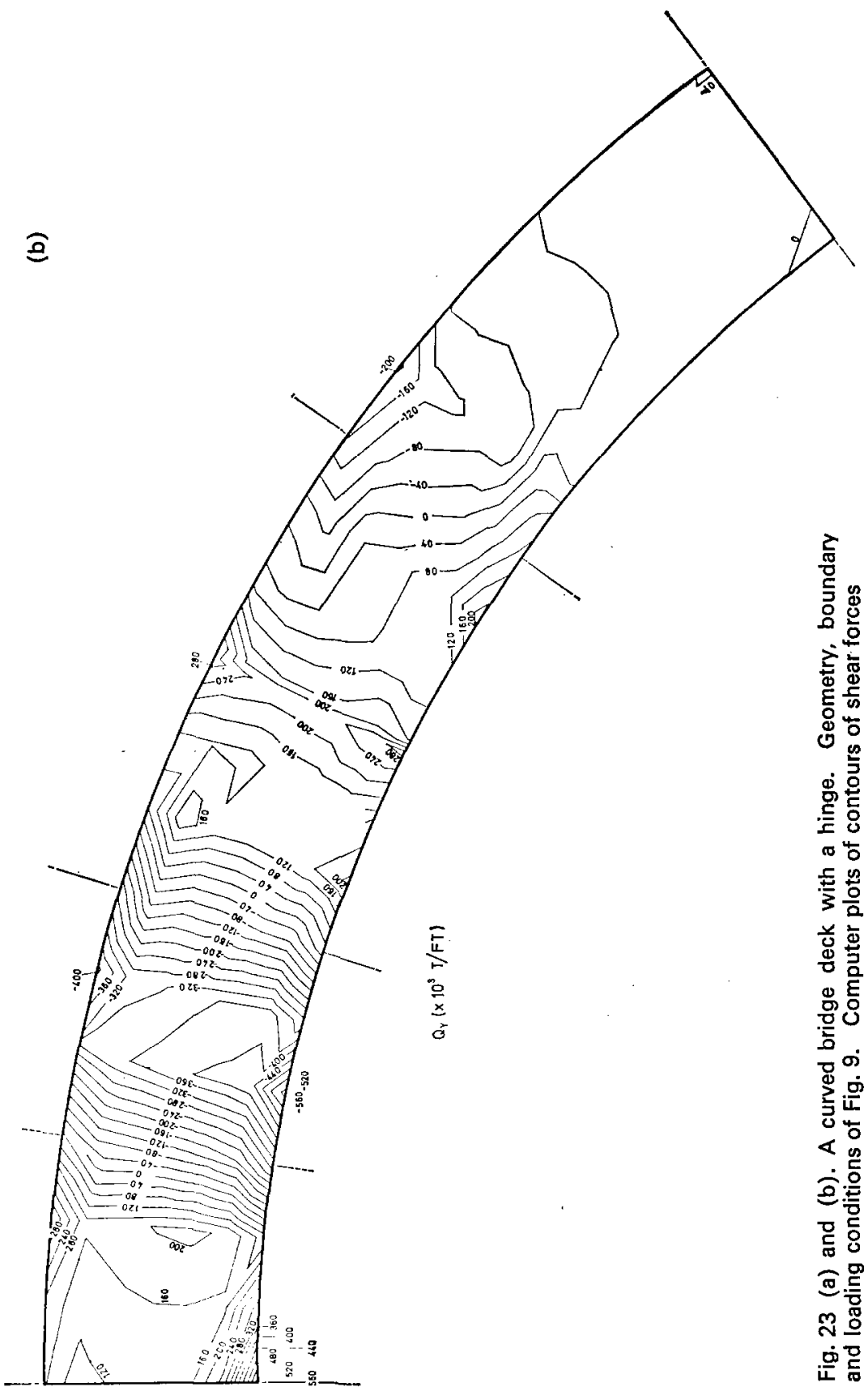


147. It would seem that with the more refined analytical techniques now available careful consideration must be given at the analysis stage to the exact support conditions expected. If incorrect assumptions are made as in the analysis described in the Paper, quite considerable errors can arise as shown in Fig. 8(a).

\section{Mr P. T. K. Lim and Dr J. C. Chapman}

The successful application of numerical methods depends on the idealization of the structure and on the choice of mesh size, which must be small enough to give the required accuracy yet large enough not to demand an exorbitant use of computer time. The necessary mesh size depends on the strain or curvature gradients found in a particular problem and good judgement in this respect depends on direct or vicarious experience of a range of problems. The Paper serves the valuable purpose of making available some of the Authors' experience of flexural problems.

149. We have considered the integral-column head as a two dimensional problem, particularly in respect of the rotational stiffness afforded by the slab, using triangular elements. The problem was solved by assuming the flexural rigidity of the slab to be greatly increased over the column area, and by applying linearly varying normal forces to the elements in this area. A similar result was obtained by applying a couple to one of the nodes in the column area. An alternative solution was obtained by taking a rigid area to which a rotation was applied, but this solution is less convenient since the associated moment must then be found by addition of the internal slab moments. Good agreement was found with a model test.

150. We have recently compared the results of triangular element solutions for skew and curved continuous bridge decks with the results of model tests. Good agreement was found for moments and reactions. Where there are more than two reactions on a support line, the individual reactions (but not the total reaction on a line) are sensitive to the vertical stiffness assumed for the reaction. Since the support stiffness in an actual bridge will not usually be known accurately, the use of a finely graded net in the region of the reactions to increase the apparent accuracy may not be justified.

151. The triangular element has the advantage of adaptability to any plan shape, but for a given number of nodes, the accuracy is not so good as for rectangular elements. Would the Authors comment on the use of triangular elements in conjunction with rectangular elements, having regard to their non-conformity, and on the use of quadrilateral elements?

\section{Dr Cheung, Dr King and Professor Zienkiewicz}

The Authors are delighted both with the general response, illustrating the progress which has been made in the acceptance and development of the finite element processes, and in the detailed observations highlighting and simplifying points made in the Paper.

\section{Finite element types}

153. The choice of the element idealization to be used in the solution of any problem is not a straightforward matter. Assuming the elements are such that geometry of the structure can be adequately represented by the elements used, it is generally found that higher order elements with larger numbers of degrees of freedom produce an improved accuracy for a given number of total degrees of freedom. ${ }^{32}$ However, to an engineer the cost of computer time and data preparation is important and the optimum does not always lie with complex elements due to the time taken in their formulation.

154. Professor Sawko uses simple rectangular elements which for a given degree of freedom are slightly superior to the triangle. A more refined rectangle is used by Dr Baker and Mr MacMillan. Both these elements suffer from the lack of geometrical generality and as such have been rejected in our basic system. 
155. An interesting development is introduced here by Dr Jenkins and Mr Lax in the special element with a rigid corner. This element appears to be rather specialized to slab bridge problems only but should be a useful addition to the element 'armoury' of a general system. They should be congratulated on this development.

156. A program of the type described in the Paper permits incorporation of various element types and can be modified to suit 'personal' preferences. Nevertheless the particular triangular element used is such that economical and accurate solutions can be readily achieved for a variety of slab problems, not alone occurring in bridge work.

157. The question of constant versus variable thickness elements has been raised by Professor Sawko. A variable thickness triangle has in fact already been developed and used extensively. ${ }^{33}$ A slight accuracy improvement is achieved but contrary to what is suggested by Professor Sawko no difficulty (theoretical or numerical) exists in joining elements with sudden thickness changes. This indeed is often the actual situation and must be catered for in any general program.

\section{Shear deformations and orthotropy}

158. Professor Sawko makes an interesting comment pointing out the need for considering shear deformations in cases of highly cored slabs. It must be pointed out, however, that the importance or otherwise of shear deformation cannot be determined entirely on the basis of such diagrams as given in Fig. 18. The question of thickness to span ratio is an overwhelmingly important factor as is indeed known from ordinary beam theory. 'Shear' deformation may thus not be important in a vierendeel truss of large length, and may be predominant in a solid short and thick beam.

159. Clearly many bridges are used in which even without coring shear effects are important. Although it is in principle possible to modify conventional plate bending

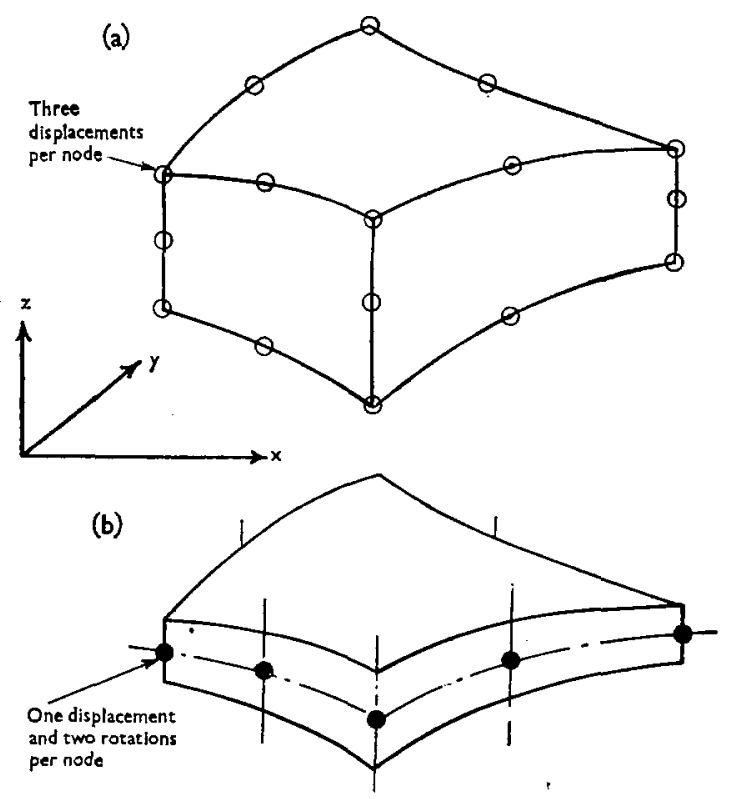

Fig. 24. A 60 degree of freedom, three dimensional curved element (a) degenerated to a 24 degree of freedom thick plate (b) 
elements to include approximately some such effects (as is suggested by Professor Sawko) the Authors prefer a different approach. A curved quadrilateral element has been 'degenerated' from a full three dimensional element and can be used economically for such problems. Outline of the process is given in references 32 and 34 and a special paper dealing with application to bridge problems is now being prepared (Fig. 24).

160. With regard to orthotropy the Authors were interested to learn about the rather small effect of cellular sections on orthotropic constants from Dr Baker and Mr MacMillan. Perhaps the words 'highly anisotropic' were not applicable here.

161. Model tests for determination of anisotropy constants are obviously useful but as mentioned by Professor Sawko numerical analysis can also yield appropriate answers. Three dimensional analysis is nevertheless necessary for determination of constants in direction parallel to coring and for twist effects. For transverse bending only plane strain conditions prevail.

\section{Cumberland Basin Bridge}

162. Several contributors (Dr Baker, Mr MacMillan, Mr Best and Mr West) refer to this example. Data for this problem were taken from reference 14 and the support elasticity was not included as relevant information was not given in the original paper and also comparable analytical work of Morley ${ }^{15}$ was a safer basis for assessment. As indicated in the Paper, elastic supports (if their properties are known) are dealt with easily.

163. Also a comparison in the Cumberland Bridge case involved only the reactions as no strain measurements were available to the Authors to compare bending moments.

\section{Column heads}

164. Further work has been done in this connexion since the presentation of the Paper and will be reported in detail elsewhere. Indeed a three dimensional study of this and other details on the same lines as suggested by $\mathrm{Mr}$ Thorpe, $\mathrm{Mr}$ Lim and Dr Chapman is in progress.

165. The treatment of column heads in a two-dimensional manner described by Mr Lim and Dr Chapman by increasing the slab stiffness over column area is now used as standard. This reproduces quite adequately the general slab behaviour and moments near the column edge but warning should be given not to place any significance to moments obtained within the column area. As a result of the work now in progress it is hoped to derive data for predicting stresses over column heads from slab moments in the vicinity.

\section{Non-concentric beams}

166. This frequently encountered situation is commented on by Mr Kretsis, Dr Jenkins and Mr Lax. Currently programs are available for dealing with such problems (in reference 35 this is applied to a general shell problem). As illustrated in Fig. 25 triangular elements are again used. Unfortunately six in place of three degrees of freedom have to be used to solve such problems and therefore the cost is rather large. A research project is currently in progress to determine whether by use of suitable co-planar beam substitutes adequate answers can be obtained at lesser cost. If beams are relatively small as at the Castleton example, the moments predicted in them should be reasonable if allowance is made for 'flange effect'.

\section{Grillage analogy}

167. Professor Sawko re-opens the vexed subject of the use of substitute structures. It has long been the practice of engineers to use grillages of different kinds to solve continuum, plate and shell problems if means were available for solving the former but not the latter. Vice versa, just as frequently, continuum, plate or membrane or shell solutions of an exact or approximate nature have been used to solve situations 


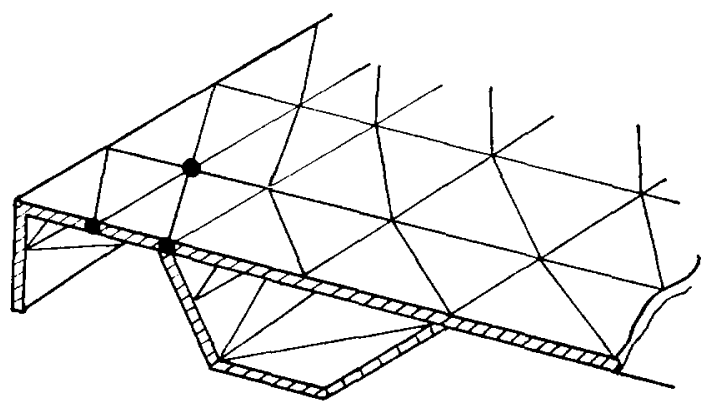

Fig. 25. A deep beam or hollow section bridge treated as a special core at a general shell solution by triangular element (see ref. 35)

where in fact a lattice or grillage existed. Solutions can be achieved by both means utilizing the tool kit most readily available.

168. In the type of program described in the Paper both plates and beams can be dealt with directly. Thus if a real situation were that of a grillage it would be solved as such. Similarly a plate would be treated as a plate. Computationally the effort is precisely the same for a given nodal subdivision and thus there is a considerable waste of labour and there are certainly no gains in accuracy in desiring a physical approximation before proceeding with the solution of the main problem. If, however, a user of a finite element program is predisposed to indulge in this indirect approach he is always at liberty to do so. Thus the grillage analogue can, if desired, be considered as a 'sub-set' of the more general approach.

\section{Practical application}

169. Mr Parekh and Mr Anderson have brought out already the importance of 'streamlining' input and providing simple programs for processing output of finite element bridge programs. This work carried out with support of the Ministry of Transport is typical of a general approach possibility in use of complex programs by the practising engineer. At the expense of sacrificing generality the data preparation work for a narrow class of problems can be condensed to a minimum. Thus the IPUT program for instance limits the user to bridges of constant width and prescribed plan curvature. Fortunately the user demand for such structures is sufficient to justify a special simple treatment here. If, however, a bifurcating curved slab with plan openings had to be treated, the user would have to return to the most general but more complex input.

170. Similarly various specialized output treatments are necessary to deal with the final answers in the manner suiting the design engineer's needs. Thus special 'tail packages' may be necessary for reinforced or prestressed slabs, and it is possible at this stage to visualize an optimizing output program which would terminate in complete reinforcement specifications. Whether such programs will in fact be written depends much on the magnitude of the need.

\section{References}

20. SAwko F. and COPE R. J. The use of finite elements for the analysis of right bridge decks. International Symposium on the Use of Electronic Digital Computers in Structural Engng. Newcastle-upon-Tyne, June 1966.

21. SAWKo F. Recent developments in the analysis of steel bridges using electronic computers. British Constructional Steelwork Association Conference, London, June 1968. 
DISCUSSION

22. SAwro F. and Cope R. J. The analysis of skew bridge decks-a new finite element approach. To be published in Struct. Engr.

23. HaRris G. Bending of beams with perforated webs. Third year project report, University of Leeds, 1967.

24. FAY C. Bending of beams with perforated webs. Third year project report, University of Leeds, 1967.

25. SAwKo F. Bridge deck analysis-electronic computers versus distribution methods. Civ. Engng Pub. Wks Rev., 1965 (April) 534-538.

26. ButLin G. A. The finite element method applied to plate flexure. $\mathrm{PhD}$ thesis, University of Cambridge, 1966.

27. Best B. C. and West R. Private communication, 1968.

28. PAReKh C. PROGRam (BaPs) - bridge and plate system. Computer program report No. 6, University of Wales, Swansea, April 1968.

29. Parekh C. PROGRam (IPUT) - bridge input program. Computer program report No. 7, University of Wales, Swansea, May 1968.

30. Phillits D. PROGRam (oput)-bridge output program. Computer program report No. 8, University of Wales, Swansea, May 1968.

31. ZIENKIEWICZ $\mathrm{O}$. C. and CHEUNG Y. K. The finite element method in structural and continuum mechanics. McGraw-Hill, London, 1967.

32. Irons B. M. and ZIENKIEwICZ O. C. The isoparametric finite element systema new concept in finite element analysis. Prof. Conf. joint British Comm. Strain Analysis. Royal Aeronautical Society, London, 1968.

33. Anderson R. G. et al. Vibration and stability of plates. Int. J. Solids Struct., 1968. To be published.

34. Ahmed S., Irons B. M. and Zienkiewicz O. C. Curved, thick shell elements. 2nd Conference on matrix methods in structural mechanics. Air Force Inst. Techn, Ohio. Oct. 1968.

35. Zienkiewicz O. C., Parekh C. and King I. P. Arch dams analysed by a linear finite element shell solution program. Proceedings of symposium on arch dams. Institution of Civil Engineers, London. Dec. 1968. 